\title{
Estudio de la evolución y técnica de regulación de la corriente presistente en anillos superconductores de YBCO
}

\author{
I. QUELLE', H. GONZÁLEZ-JORGE², L. ROMANí' y G. DOMARCO'1 \\ 'Dpto. Física Aplicada, Universidad de Vigo, Facultad de Ciencias, As Lagoas, 32004 Ourense \\ 2Dpto. de Temperatura y Humedad, Laboratorio Oficial de Metroloxía de Galicia, \\ Parque Tecnolóxico de Galicia, San Cibrao das Viñas s/n, 32901 Ourense
}

\begin{abstract}
En este trabajo se realiza un estudio de la evolución de corriente en función de la densidad de vórtices atrapados y se presenta una técnica para regular la corriente partiendo del valor crítico, en anillos superconductores de YBCO. Para generar una corriente persistente en los anillos se utiliza un método de tipo inductivo, denominado field cooling, que combinado con campos magnéticos en sentido contrario al inductor permite disminuir la densidad de vórtices atrapados y se logra también disminuir el decaimiento de corriente. Una vez que se ha inducido la corriente crítica en los anillos una zona muy localizada de éstos se calienta hasta que el superconductor alcanza el valor deseado de corriente, lo cual se comprueba mediante la medida de campo magnético con una sonda Hall.
\end{abstract}

Palabras clave: superconductores, propiedades eléctricas, propiedades térmicas, anillo YBCO

Study and technique for controlling the persistent current in the YBCO superconducting rings.

In this work, a study of the relation between the density of trapped vortices and the current evolution and a technique for controlling the current from its critical value in YBCO rings, are performed. To generate the persistent current in the rings, an inducting technique called field cooling is used. Combining the field cooling technique with magnetic fields in opposite direction to the inducting one, the density of trapped vortices decreases and the current decay also decreases. Then, a part of the sample is heated to decrease the current to the desired value, which is checked using a Hall sensor.

Keywords: superconductors, electric properties, thermal properties, YBCO ring.

\section{INTRODUCCIÓN}

Se conoce desde hace años la capacidad que tienen los anillos superconductores para transportar una corriente persistente generada en los mismos por un procedimiento inductivo (1-2). Dicho procedimiento consiste en lo siguiente: Se enfría el anillo superconductor en presencia de un campo magnético paralelo al eje del anillo, desde la temperatura ambiente hasta una temperatura menor que su temperatura crítica (temperatura por debajo de la cual alcanza el estado de resistencia cero). Una vez que la temperatura es inferior a la crítica, se suprime el campo magnético y el superconductor responde inductivamente a la variación de flujo generando una corriente persistente, de forma que el flujo magnético a través del agujero del anillo permanezca constante.

Para la inducción de corrientes elevadas en los superconductores se han venido utilizando grandes bobinas que dificultaban su aplicación práctica. Recientemente, se ha desarrollado un método que consiste en enfriar el anillo superconductor emplazado en un núcleo de hierro, que es magnetizado por una pequeña bobina (3). Debido a la alta permeabilidad magnética del hierro, aun empleando pequeñas bobinas de excitación se alcanzan grandes campos magnéticos en el agujero del superconductor. De esta forma se inducen corrientes elevadas de forma muy sencilla.
Lacorrientepersistenteinducidaen anillossuperconductores de tipo II sigue un decaimiento de tipo logarítmico acorde al modelo de Anderson $(4,5)$ :

$$
\mathrm{I}=\mathrm{I}_{0}\left[1-\frac{\mathrm{k}_{\mathrm{B}}{ }^{\top}}{\mathrm{U}_{0}} \operatorname{Ln}\left(1+\frac{\mathrm{t}}{\mathrm{t}_{0}}\right)\right]
$$

donde $k_{B}$ es la constante de Boltzman, $T$ la temperatura de trabajo, $U_{0}$ la barrera de potencial para los vórtices y $t$ el tiempo de duración del experimento.

La causa del decaimiento de dicha corriente se debe al movimiento irreversible de vórtices debido a la fuerza de Lorentz (6). La disminución de la pendiente de decaimiento puede conseguirse de dos formas: ralentizando el movimiento de vórtices inducidos, mediante la generación de centros de pinning en el material, o disminuyendo su densidad.

En éste trabajo se plantean dos objetivos:

- Disminuir la cantidad de vórtices atrapados combinando técnicas de tipo inductivo.

- Se presenta una técnica que permite la regulación de la corriente inducida en el superconductor a partir de su corriente crítica. Ésta técnica se basa en la utilización de un pequeño 
calefactor dispuesto en la superficie del superconductor, y una sonda Hall (7) para el control del valor de la corriente.

\section{EXPERIMENTAL}

\section{1.-Muestras}

Las muestras utilizadas en este trabajo son anillos superconductores de YBCO obtenidos a partir de pastillas crecidas por top seeding melt grown, procedentes del Institut für Materialphysik de la Universidad de Göttingen (Alemania), que fueron perforadas con una broca de diamante-cobre (8). Las dimensiones de los anillos superconductores utilizados, YBCO1, YBCO2, YBCO3 e YBCO4, se muestran en la Tabla I, donde $R_{1}$ y $R_{2}$ son los radios interior y exterior, y $\mathrm{H}$ la altura. Los valores de la corriente crítica en los anillos se muestran también en la Tabla I.

TABLAI.CORRIENTECRÍTICAYDIMENSIONESDELOSANILLOSSUPERCONDUCTORES UTILIZADOS, YBCO1, YBCO2, YBCO3 E YBCO4. I CORRIENTE CRÍTICA, R Y R R RADIOS INTERIOR Y EXTERIOR, H ALTURA.

\begin{tabular}{|c|c|c|c|c|}
\hline & YBCO1 & YBCO2 & YBCO3 & YBCO4 \\
\hline$I_{C}(A)$ & 4400 & 4000 & 2600 & 4600 \\
\hline $\mathrm{R}_{1}(\mathrm{~mm})$ & 5 & 5 & 5 & 5 \\
\hline $\mathrm{R}_{2}(\mathrm{~mm})$ & 14 & 14 & 10 & 14 \\
\hline $\mathrm{H}(\mathrm{mm})$ & 10 & 10 & 8 & 10 \\
\hline
\end{tabular}

\section{2.- Inducción de corriente}

Para generar una corriente persistente en los anillos se utiliza un método de tipo inductivo, denominado field cooling. Esta técnica consiste en aplicar un campo magnético en la dirección del eje del anillo a una temperatura superior a la crítica Tc (debido a la resistencia del anillo la corriente inducida es inmediatamente disipada). A continuación, el superconductor se enfría a campo magnético constante y se retira el campo cuando la temperatura es inferior a la crítica. La variación de flujo magnético en el orificio del anillo superconductor provoca la inducción de una corriente persistente en el mismo. Dicha corriente genera un campo magnético del mismo módulo y sentido que el inductor. La evolución de la corriente se mide situando una sonda Hall en el centro del anillo.

Para inducir la corriente persistente en el superconductor se han utilizado dos técnicas:

En la primera, se utiliza un circuito ferromagnético cerrado, magnetizado mediante una pequeña bobina, que atraviesa el centro del anillo superconductor (9) (Figura 1a). La corriente que necesita la bobina del circuito ferromagnético para la inducción de la corriente crítica superconductora es de $0.4 \mathrm{~A}$ para YCBO1 y 0.3 A para YBCO2. Los campos magnéticos que se generan en el orificio de los anillos son de $250 \mathrm{mT}$ y $200 \mathrm{mT}$, respectivamente.

En la segunda, se utiliza un método de inducción combinado. Consiste en un circuito ferromagnético cerrado (campo magnético $B_{F}$ en Figura $1 \mathrm{~b}$ ), magnetizado también mediante una pequeña bobina como en el caso anterior, y dos grandes imanes de piezas polares paralelos al eje del anillo (campo magnético $B_{I}$ en Figura $1 b$ ). $B_{S}$ indica el campo magnético que incide sobre la pared del superconductor. Para lograr con ésta técnica la inducción de corriente crítica y la disminución de la densidad de vórtices (disminución del decaimiento de corriente en la muestra), los campos magnéticos deben ser de la misma dirección y distinto sentido, y tener las siguientes características:

- Distinta magnitud en el centro del anillo superconductor, de modo que no se anulen y el campo resultante induzca en el anillo la corriente crítica.

- Se deben anular sobre la pared del superconductor (campo magnético $B_{S} \rightarrow 0$ en Figura $1 b$ ) para minimizar la densidad de vórtices inducidos (1).
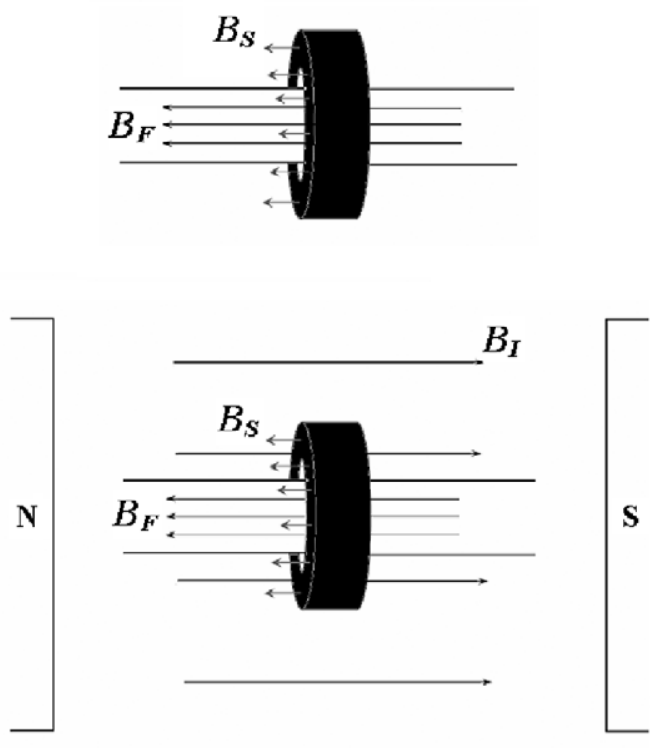

(b)

Fig. 1- Técnicas de inducción (a) utilizando un circuito ferromagnético cerrado que atraviesa el centro del anillo, magnetizado con una pequeña bobina, (b) combinando el circuito ferromagnético magnetizado y dos grandes imanes de piezas polares paralelas al eje del anillo, que generan campos de sentido contrario. $\mathrm{B}_{\mathrm{S}}$ campo en el cuerpo del anillo superconductor (flujo atrapado), $\mathrm{B}_{\mathrm{I}}$ campo producido por los imanes y $\mathrm{B}_{\mathrm{F}}$ campo del circuito ferromagnético.

Para el YBCO1 se hace circular una corriente en la bobina de 4 A y para el YBCO2 de $3 \mathrm{~A}$, que generan campos $750 \mathrm{mT}$ y $650 \mathrm{mT}$ en el orificio de la muestra. El campo de los imanes es de $300 \mathrm{mT}$ (en el núcleo) para la inducción de corriente en YBCO1 e YBCO2. Se han utilizado corrientes en la bobina 10 veces superiores que en la inducción de corriente sólo con el circuito ferromagnético. De esta forma, el campo generado supera suficientemente al campo de los imanes en el centro del superconductor y se alcanza la corriente crítica. La anulación del campo en las paredes de la muestra superconductora se obtiene regulando la distancia de los imanes.

\section{3.- Regulación de corriente}

El dispositivo térmico utilizado para la regulación de la corriente persistente que circula por un anillo superconductor se basa en la propiedad que tienen los materiales superconductores por la que, aún permaneciendo a una temperatura inferior a la crítica, la corriente que puede circular por los mismos es función de la temperatura a la que están sometidos (10-11).

Como dispositivo térmico para el calentamiento local del anillo superconductor (Figura 2a), en contacto con éste y arrollado sobre él, se realiza un bobinado de hilo metálico de resistencia elevada. 


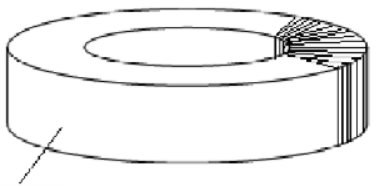

(a)

(I)

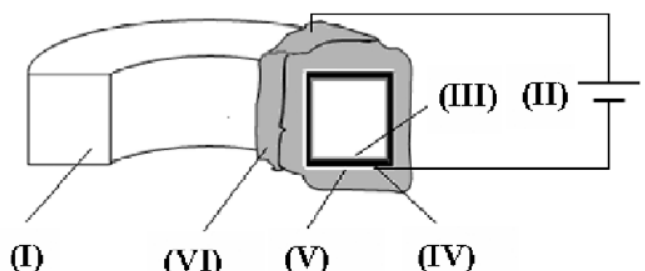

(b)

Fig. 2- (a) Anillo superconductor (b) Corte en sección del superconductor y del elemento calefactor.

En la Figura $2 b$ se ve un corte en sección del superconductor (I) y del elemento calefactor, alimentado con una fuente de corriente continua (II). Como calefactor se utiliza un hilo metálico de constantán, de resistividad $45 \cdot 10^{-8} \Omega \cdot \mathrm{m}$ a la temperatura del nitrógeno líquido. Previamente al embobinado del hilo, se deposita sobre el anillo una grasa de contacto térmico Apiezon tipo N (III). Sobre la grasa se realiza el bobinado anti-inductivo de hilo de constantán (IV), de modo que los campos magnéticos generados al circular la corriente por el hilo se anulan, evitando la inducción de cualquier corriente en el anillo superconductor. La resistencia de dicho embobinado se mide para las muestras YBCO3 e YBCO4 y se obtienen $27.9 \Omega$ y $22.8 \Omega$, a la temperatura del nitrógeno líquido. Finalmente, la resistencia se aísla con una fina capa de teflón $(\mathrm{V})$ y una capa más gruesa de poliuretano (VI), para evitar el contacto directo con el nitrógeno líquido.

El primer paso consiste en generar la corriente crítica en las muestras, para lo que se utiliza el método de tipo inductivo de enfriamiento con campo. La corriente que necesita la bobina del circuito magnético para la inducción de la corriente crítica superconductora se calcula utilizando la ecuación para un circuito magnético cerrado (12) y se obtienen $0.3 \mathrm{~A}$ para YCBO3 y 0.4 A para YBCO4. Para asegurar con mayor certeza el valor de corriente crítica, la corriente en la bobina se sobredimensiona un factor 10 , siendo entonces las corrientes utilizadas 3 A y 4 A para YBCO3 e YBCO4, respectivamente.

Una vez inducida la corriente crítica en las muestras, ésta se comprueba utilizando una sonda Hall situada en el centro de la muestra y con la ley de Biot y Savart:

$$
B_{s}=\frac{\mu_{0} l_{s}}{\sqrt{h^{2}+\left(R_{1}+R_{2}\right)^{2}}}
$$

donde $\mathrm{B}_{\mathrm{S}}$ es el campo del superconductor, $\mathrm{I}_{\mathrm{S}}$ la corriente superconductora y $\mu_{0}$ es la permeabilidad magnética del vacío (9).

De esta forma, se obtienen valores de campo magnético de $192 \mathrm{mT}$ para YBCO3 y $269 \mathrm{mT}$ para YBCO4, que dan como resultado corrientes de 2601 A para YBCO3 y 4603 A para YBCO4.

Una vez inducida la corriente crítica en los anillos, el objetivo de este trabajo consiste en disminuir ésta hasta el valor deseado. Para ello, una zona muy localizada del anillo se calienta con el dispositivo térmico, haciendo circular por éste una corriente. La temperatura local de la muestra en la superficie de contacto aumenta, disminuyendo la corriente que puede pasar por esa zona, y a su vez la que pasa por el conjunto del anillo. La disminución de corriente se comprueba en cada instante mediante la sonda Hall situada en el centro del anillo.

\section{RESULTADOS}

En las Figuras 3 y 4 pueden verse las curvas de decaimiento de la corriente en los anillos superconductores para los dos métodos de inducción. Los valores de corriente en los anillos superconductores están normalizados respecto a la corriente crítica, y se representan frente al logaritmo del tiempo (modelo teórico de decaimiento propuesto por Anderson).

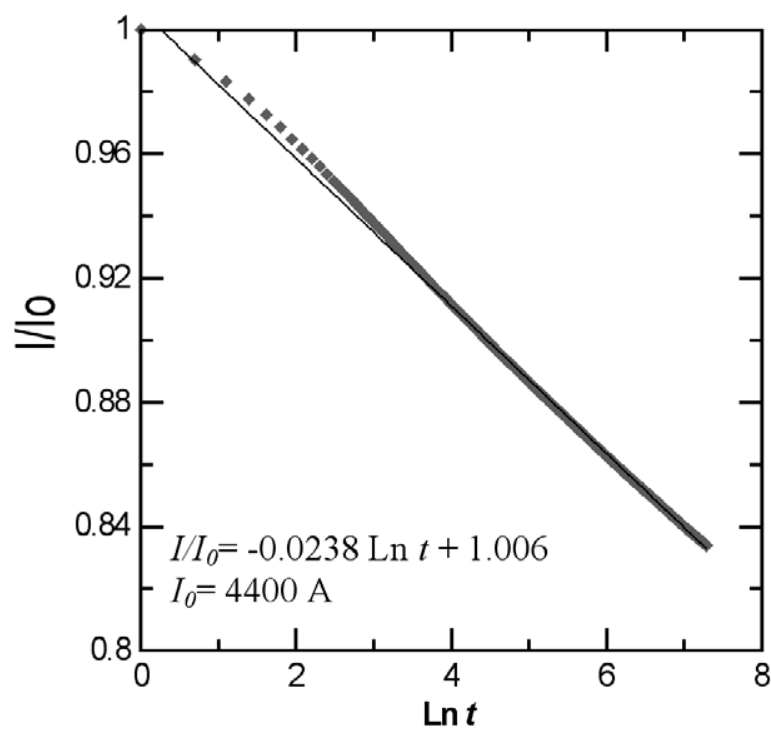

(a)

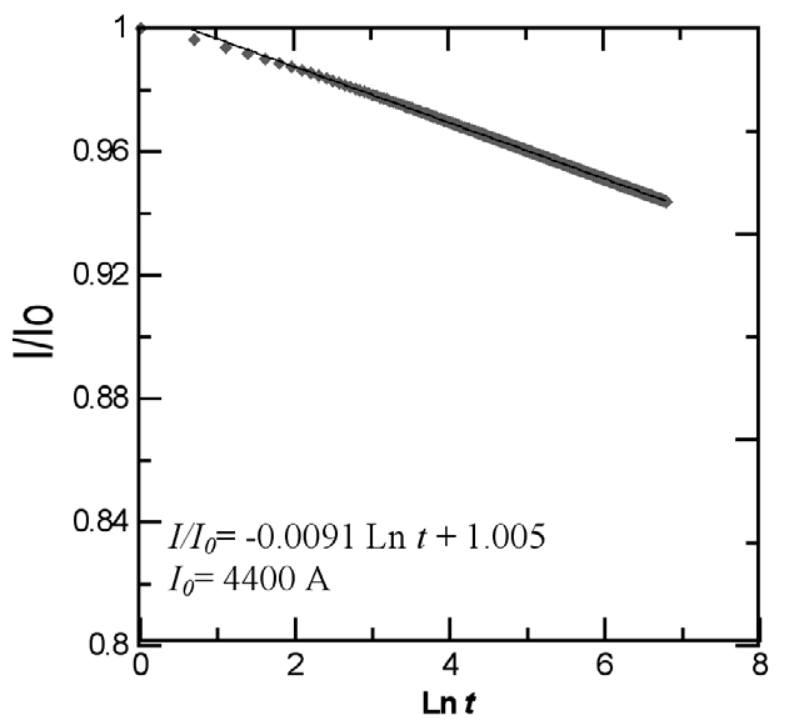

(b)

Fig. 3- Decaimiento de la corriente en el anillo superconductor YBCO1, con corriente crítica $4400 \mathrm{~A}$. La corriente se indujo: (a) con un circuito ferromagnético cerrado magnetizado con una pequeña bobina, (b) con un circuito ferromagnético cerrado magnetizado con una pequeña bobina y dos imanes de grandes dimensiones, que generan campos de sentido contrario. I es la corriente en el anillo, $\mathrm{I}_{0}$ es la corriente inicial y t el tiempo. 
En las Figuras 3a y 4a se observa el decaimiento de la corriente en los anillos superconductores YBCO1 e YBCO2, con corrientes críticas de 4400 A y 4000 A, donde dicha corriente se indujo con un circuito ferromagnético cerrado magnetizado con una pequeña bobina. La pendiente de decaimiento de la corriente es -0.0238 para la muestra YBCO1 y -0.0221 para la muestra $\mathrm{YBCO} 2$.

En las Figuras $3 \mathrm{~b}$ y $4 \mathrm{~b}$ se observa el decaimiento de corriente para los mismos anillos, en los que se obtiene una pendiente de decaimiento de corriente -0.0091 en ambos casos. En este caso se ha utilizado para la inducción de corriente crítica la técnica que combina imanes y circuito ferromagnético. Como se puede observar, utilizando esta técnica se logra una disminución de la pendiente de decaimiento superior al 50\% debida a la disminución en la densidad de vórtices en la pared del superconductor.

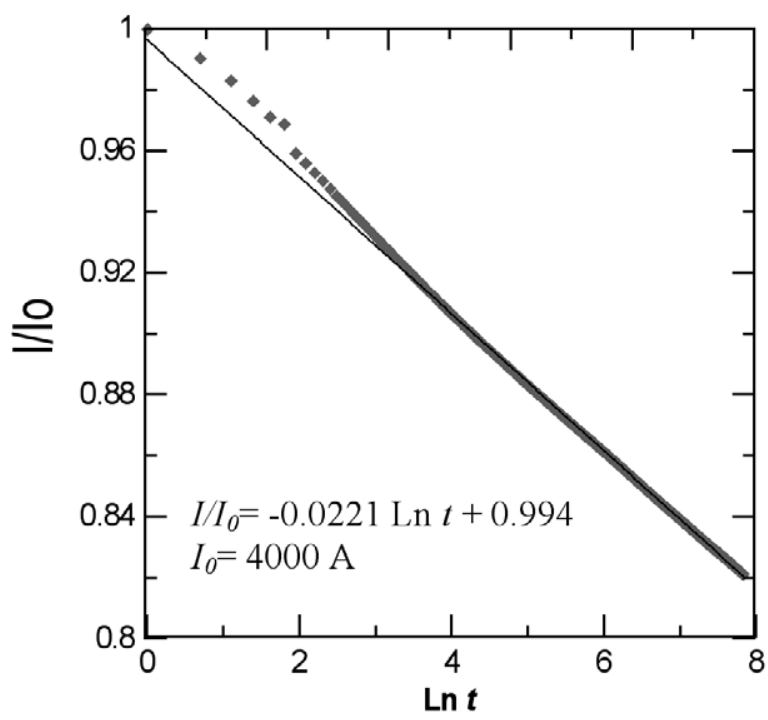

(a)

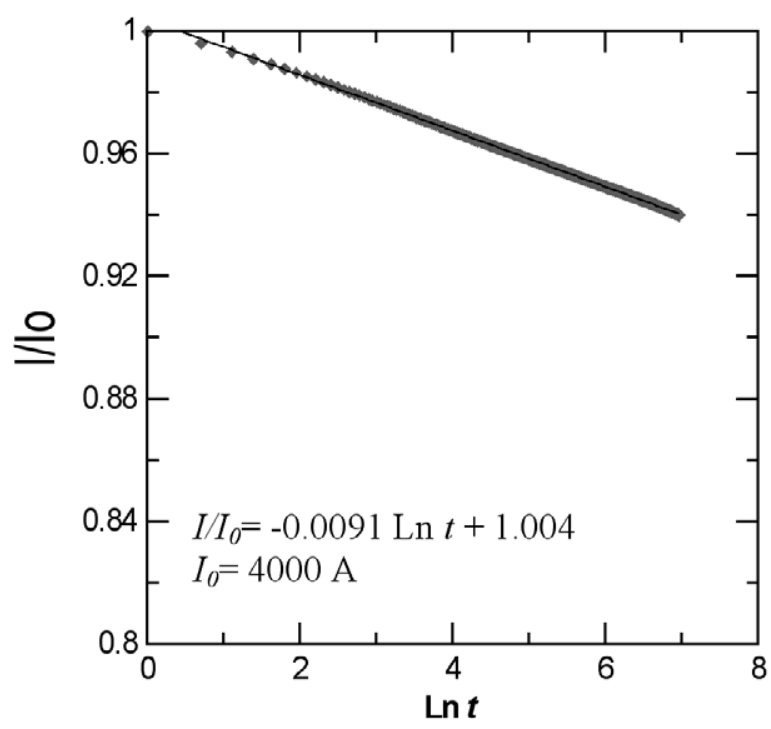

(b)

Fig. 4- Decaimiento de la corriente en el anillo superconductor YBCO2, con corriente crítica $4000 \mathrm{~A}$. La corriente se indujo: (a) con un circuito ferromagnético cerrado magnetizado con una pequeña bobina, (b) con un circuito ferromagnético cerrado magnetizado con una pequeña bobina y dos imanes de grandes dimensiones, que generan campos de sentido contrario. I es la corriente en el anillo, $\mathrm{I}_{0}$ es la corriente inicial y t el tiempo.
Las Figuras 5 y 6 muestran los resultados obtenidos para el control de la corriente en las muestras YBCO3 e YBCO4, respectivamente.

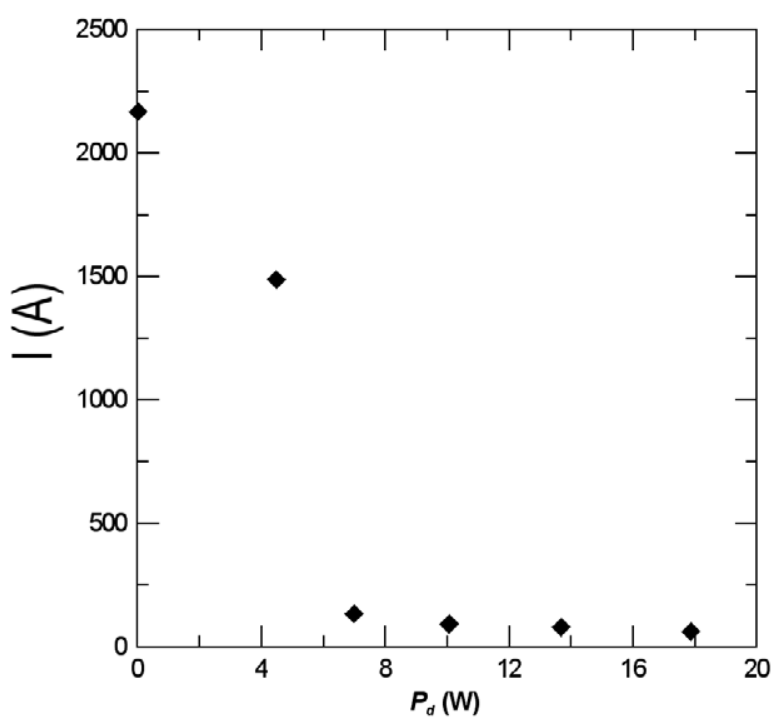

(a)

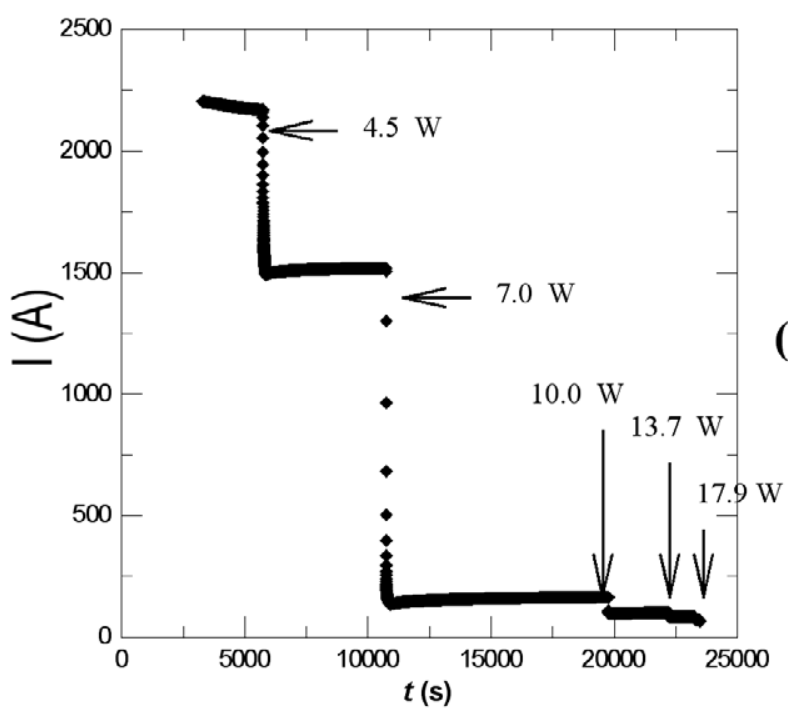

Fig. 5- Anillo superconductor YBCO3. (a) Corriente que circula en la muestra frente a potencia disipada en la resistencia usada como dispositivo térmico. (b) Corriente en la muestra frente a tiempo de la experiencia, indicada la potencia disipada en la resistencia para cada calentamiento. I es la corriente en la muestra, $\mathrm{P}_{\mathrm{d}}$ es la potencia disipada en la resistencia y t el tiempo de la experiencia.

En las Figuras 5a y 6a se representa la corriente obtenida en función de la potencia disipada. Se observa que sigue un comportamiento similar a la corriente crítica en función de la temperatura (2), lo que corrobora la capacidad del método.

En las Figuras 5 b y 6 b se representa la corriente frente al tiempo y se indica la potencia disipada en la resistencia para la disminución de la corriente. En dichas figuras se puede observar el rápido descenso de la corriente en las muestras producido por el calentamiento local.

Resulta importante reseñar que, transcurridos unos pocos segundos, la disminución de corriente en las muestras es proporcional a la potencia y no a la energía disipada. Es decir, 
se acaba produciendo un equilibrio entre la temperatura que alcanza la muestra, la potencia constante disipada sobre ella y la temperatura constante del nitrógeno líquido. Una vez alcanzado este equilibrio la temperatura de la muestra no aumenta más y la corriente que circula por la misma ya no disminuye.

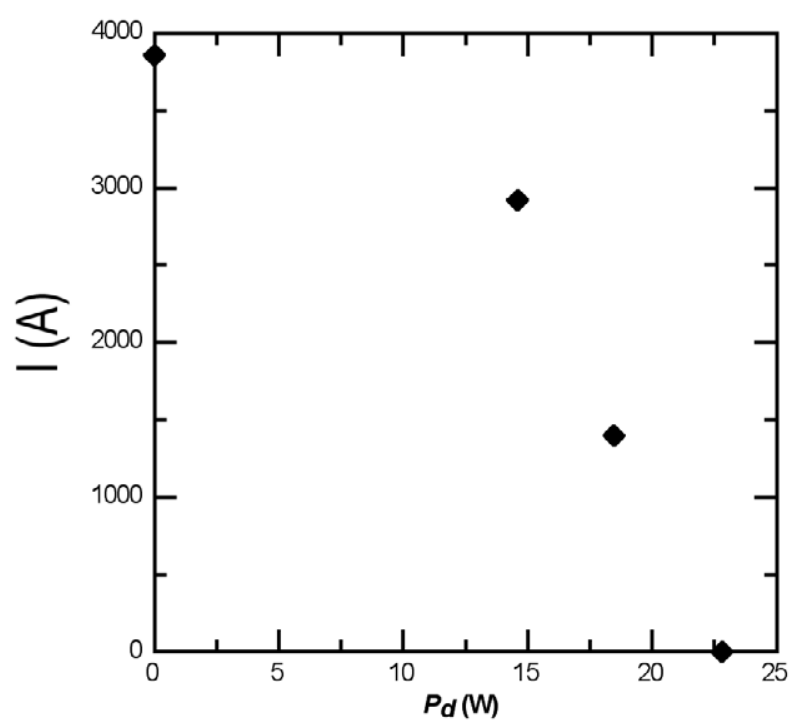

(a)

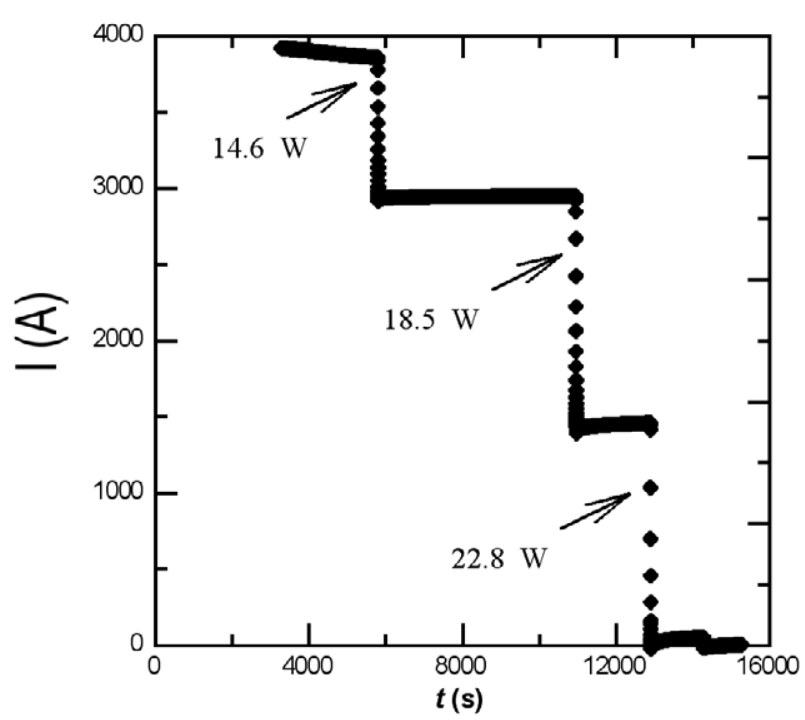

(b)

Fig. 6- Anillo superconductor YBCO4. (a) Corriente que circula en la muestra frente a potencia disipada en la resistencia usada como dispositivo térmico. (b) Corriente en la muestra frente a tiempo de la experiencia, indicada la potencia disipada en la resistencia para cada calentamiento. I es la corriente en la muestra, $\mathrm{P}_{\mathrm{d}}$ es la potencia disipada en la resistencia y t el tiempo de la experiencia.

Con los datos aquí obtenidos, se puede ahora realizar una calibración del procedimiento, de forma que se obtenga una ecuación de ajuste entre la potencia disipada y la corriente superconductora. Con esto datos, ya se puede obtener a continuación cualquier corriente superconductora a partir de la corriente crítica.

En definitiva, esta técnica abre una puerta al control de la corriente en anillos superconductores en los que se induce la corriente crítica, mostrándose como muy sencilla y eficaz.

\section{AGRADECIMIENTOS}

Este trabajo ha sido financiado por la Dirección General de Innovación de la Consellería de Innovación e Industria de la Xunta de Galicia mediante el proyecto $\mathrm{n}^{\circ}$ PGIDIT05DPI38301PR.

\section{BIBLIOGRAFÍA}

1. H. González-Jorge, J. Peleteiro, E. Carballo, and G. Domarco, Physica C, 433 (1-2) 132-138 (2005).

2. J. Jung, I. Isaac, M.A.-K. Mohamed, Physical Review B, 48 (10) 7526-7536 (1993).

3. H. González-Jorge, E. Carballo, J. Peleteiro, and G. Domarco, Journal of Superconductivity, 18 (4) 541-544 (2005).

4. H. González-Jorge, J. Peleteiro, J. Troncoso, E. Carballo and G. Domarco, Cryogenics, 45, 135-140 (2005).

5. P. W. Anderson, Physical Review Letters, 9, 309 (1962).

6. E. Durand, Magnétostatique, Masson et Cé, Editeurs (1968).

7. E. Ramsden, Hall Effect Sensors: Theory and Applications, Advanstar Communications (2001).

8. H. González-Jorge, J. Peleteiro and G. Domarco, British Ceramic Transactions, 103 (3) 145-146 (2004).

9. H. González-Jorge, J. Peleteiro, E. Carballo, L. Romaní and G. Domarco, Applied Physics Letters, 81 (22) 4207-4208 (2002).

10. G. Domarco, A. Díaz, O. Cabeza, J. Maza, A. Pomar, C. Turrón, J. A. Vieira and F. Vidal, Supercunductivity in Spain, Programa MIDAS, edited by F. Yndurain, 215 (1993).

11. A. Díaz, A. Pomar, G. Domarco, J. Maza and Félix Vidal, Physica B, 194-196, 1933-1934 (1994).

12. John David Jackson, Classical electrodynamics, John Wiley \& Sons, Inc. (1998).

Recibido: 14.03 .07

Aceptado: 23.10.07 